\title{
Education par le numérique en Haïti : enjeux, défis et perspectives
}

\author{
Nirvah JEAN-JACQUES et Bonel OXINÉ, Université d'Etat d'Haïti
}

\section{Introduction}

La révolution des technologies de l'information et de la communication (ci-après TIC) n'a point tardé à faire son apparition en Haïti ${ }^{1}$ dès ses débuts dans le monde entier. Et leur introduction, accueillie de manière dynamique et célère par le grand public, a pris de court les organismes administratifs de l'État peu préparés à les utiliser et à en réguler les conditions de distribution et d'usage : «jamais on n'a constaté une si grande différence entre la constance de temps du progrès technique et celle de son acceptation psychosociale, qui correspond à la d'une génération, environ trente ans »(Blamont 96). Ce constat de J. Blamont correspond avec exactitude à l'engouement de plus d'un pour les TIC. Pourtant, cet engouement des premières années autorisait tous les espoirs notamment pour impulser de toute part l'accès au savoir et la qualité des apprentissages (Hess et Saxberg) comme à travers l'ensemble du système éducatif haïtien. L'Internet, créé en 1991, croît aujourd'hui en doublant tous les six mois (Blamont 91) et est considéré comme le «produit phare ». Il s'en est suivi entre les organismes régulateurs nationaux et les fournisseurs de services une laborieuse et épineuse décennie de «difficultés à répétition enregistrées dans le secteur qui ne doivent pas être considérées comme l'effet du hasard, mais plutôt comme la conséquence d'un système inadéquat »(Noël). Par ailleurs, la démocratisation progressive des services d'Internet individuels et la pénétration massive de la téléphonie mobile au cours de la dernière décennie du vingtième siècle ont créé et entretenu une fièvre généralisée qui s'est traduite par une large diversité de signes : l'accroissement de la demande de services de téléphonie et d'internet (Blamont) à travers diverses zones géographiques d'Haïti, la mise en place de réseaux de transmission de données, la formation d'associations et de consortiums institutionnels pour l'établissement du «domaine ht» et la défense de leur droit de gestion dudit domaine, l'accroissement des demandes de licences d'exploitation, la multiplication des cybercafés, le développement de logiciels, l'ouverture de programmes de formation à leur utilisation dans de nombreuses entreprises. En surcroît de leur intégration dans tous les secteurs (administration privée et publique, santé, finances, Archives nationales, bibliothèques communales mises en réseau), les Technologies de l'Information et de la Communication ont été fortement promues dans le secteur d'éducation, notamment à travers des efforts de 
l'Université d'Etat d'Haïti et de nombreux organismes nationaux et internationaux publics et privés ainsi que d'universités étrangères et tant d'autres, par de nombreuses initiatives conjointes comme l'équipement de campus numérique, l'installation de laboratoires d'informatique dans des écoles, le lancement de formation de techniciens en formation à distance, etc., autant d'activités visant à promouvoir en Haïti l'utilisation des TIC à l'école. À l'heure actuelle, la mise en place de laboratoires d'informatique dans les écoles et l'offre de cours d'informatique sont monnaie courante. Bien que se soit maintenu l'enthousiasme généré dans le secteur éducation pour l'utilisation des ordinateurs et l'accès à l'internet pour la communication, l'emploi des TIC au service de l'accès au savoir ainsi qu'au développement de capacités diverses d'apprentissage guidé ou autonome ne s'est pas généralisé (Noël 14) au même rythme que l'utilisation des réseaux sociaux par exemple.

\section{Contexte et méthodologie}

Cette contribution prendra appui notamment sur les initiatives des cours en ligne mis sous pied par des organismes haïtiens, dont certains d'entre eux partent d'une vision à aboutir à une éducation de qualité en Haïti en offrant aux jeunes haïtiens, n'ayant aucune chance à bénéficier d'une formation de qualité, la possibilité d'y parvenir par le biais des TIC (voir les cours mis en ligne sur Youtube par l'École haïtienne sans frontières, 2012), et à familiariser ces jeunes de peu de moyens financiers avec les TIC, et d'autres, d'une vision à contribuer au développement communautaire ou du système éducatif du pays par la création des cours en ligne conçus en créole afin de faciliter la participation de tous les apprenants à la construction et au partage de connaissances par le biais des TIC.

\section{Problématique}

Dans le domaine éducatif, ou plus précisément dans celui d'apprentissage, comme l'a indiqué (Godinet, cité par Siméone et al), si le « développement de réseaux technologiques ne génère pas spontanément celui des réseaux humains ", il s'avère dès lors utopique de prétendre à envisager les TIC comme voie certaine conduisant au colmatage des brèches au niveau du système éducatif d'un pays dont les faiblesses et les échecs enregistrés dans ce domaine sont énormes et visibles. Utiliser les TIC, aucun système éducatif ne saurait prétendre s'en passer mais le mieux est de penser ensemble à une transformation générale du système vers un système de qualité car «A quality system must be continuously evaluated, updated, and improved to be aligned to new developments in an educational organization. 
Therefore, the following steps are necessary. The quality system should be evaluated at least twice a year. Specifically, it should be evaluated if the quality system has led to overall improvements in the organization's performance. Furthermore, the adequacy of methods, instruments, and metrics need to be evaluated »(Journal of Educational Technology and Society 2007:11). Qu'on veuille remplacer le système éducatif haïtien par les TIC en se basant sur ses promesses et progrès rapides, comme toute autre discipline, il est une évidence que «l'intégration des TIC au sein de situations d'apprentissage nécessite et/ou développe chez les acteurs - enseignants et étudiants - des compétences qui vont bien au-delà d'une simple appropriation de fonctionnalités technologiques »(Siméone et al.). De ce point de vue, nous nous demandons dans quelles mesures les cours en ligne en contexte haïtien pourront offrir la formation de qualité à laquelle s'attend tout système éducatif. L'interaction entre l'apprenant et ses pairs, l'interaction entre l'apprenant et le professeur, et enfin l'interaction entre les savoirs ou contenus et l'apprenant nous conduisent à envisager la prise en compte des différents paramètres par lesquels l'apprenant parvient à l'acquisition et à la maîtrise des savoirs visés, ce qui exigerait une certaine autonomie chez l'apprenant, en vue de gérer et rendre fonctionnels les savoirs que lui délivrent les cours en ligne, ainsi que les compétences visées. Parlant de compétences, il nous paraît pertinent de poser comme enjeux les différents types de ressources qu'exige un MOOC dans le cadre des cours en ligne. Le MOOC se définit comme un cours en ligne possédant a minima certaines caractéristiques (structure de «cours» en ligne avec un point de ralliement ouvert et massif) (Blamont 101). Ces caractéristiques précisent les conditions pour mettre les cours en ligne et les conditions pour y accéder. En ligne, comment l'apprenant bénéficiera-t-il de toutes les ressources présentées dans un MOOC? Une ressource est « un élément statique du cours » (102). Elle est au nombre de trois : ressources d'apprentissage, ressources d'accompagnement et ressources d'animation. En dépit de l'impossibilité à la technique de remplacer l'être humain, «de nombreux discours communs présentent les technologies d'information et de communication (TIC) comme susceptibles de transformer les pratiques d'enseignement/d'apprentissage, voire les modes d'accès à la connaissance » (Blamont).

\section{Questions de l'approche}

Nos questions de l'approche sont de deux ordres. L'une porte sur les cours en ligne en contexte haïtien, et l'autre, sur les avantages dont pourrait bénéficier le système éducatif haïtien à l'heure actuelle. Utiliser les TIC ou recourir à la mise des cours en ligne pour 
colmater les brèches du système éducatif haïtien, est-ce possible ? L'école haïtienne seraitelle en passe de rater les bénéfices de la révolution technologique ? Ces préoccupations constituent la toile de fond des réflexions sur les TIC que nous faisons dans la présente contribution.

\section{Éducation, TIC et Formation à distance}

La Formation à distance se définit en tant qu' « un système de formation conçu pour permettre à des individus de se former sans se déplacer sur le lieu de formation et sans la présence physique d'un formateur. La formation à distance recouvre plusieurs modalités (cours par correspondance, e-learning) et est incluse dans le concept plus général de formation ouvertes (et à distance) », selon l'AFNOR ${ }^{2}$ citée par Danneville (19). Par ailleurs, certaines études (Siméone 9) montrent que «l'intérêt de l'élaboration et de la mise en œuvre d'un scénario d'apprentissage collaboratif via les TIC a été souligné ». Et, selon H. Godinet (cité dans Siméone), la fonction de cet intérêt est de permettre « d'anticiper les rôles et les interactions des différents participants et de définir les activités en fonction des contraintes relationnelles, techniques, temporelles, au regard des résultats attendus en terme de productions et/ou d'objectifs d'apprentissage »(Godinet). Cependant, en contexte haïtien, contrairement aux foyers des pays développés que la fusion de la télévision et de l'Internet ont tous connectés, le phénomène le plus inattendu est la connexion de ceux des pays sousdéveloppés (Blamont 96). En Haïti, combien d'établissements scolaires/universitaires, voire foyers peuvent-ils être connectés au même moment, sans trop de contraintes techniques et temporelles, pour suivre un cours en ligne, pour travailler en équipe sur une tâche ? Le décalage économique du pays par rapport aux pays développés nous rend embarrés vis-à-vis de cette interrogation. En situation d'apprentissage, il ne suffit pas de réunir une somme de connaissances souvent mal assimilées et mal accumulées par l'apprenant, mais de trouver ou de mettre en place des stratégies permettant à l'apprenant de se les approprier. Comme l'a avancé J. Dewey (49), « un être lié à d'autres êtres ne peut pas exercer ses propres activités sans tenir compte de celles d'autrui, car elles sont les conditions indispensables de la réalisation de ses propres tendances ». L'appropriation du contenu est le point de réussite de tout enseignement qui vise à développer l'autonomie chez l'apprenant. Ceci implique de la part de l'enseignant l'utilisation d'outils et de techniques permettant d'identifier rapidement le «profil d'un apprenant, sa 'gestion' préférentielle, ses stratégies de réussite et d'échec » (Trocmé-Fabre 21). Nous nous demandons dans ce cas comment une formation à distance 
peut-elle prendre en considération toutes ces dimensions en vue d'atteindre une formation de qualité, à la place d'un système éducatif défaillant. Ce dont une formation à distance doit se rendre compte, c'est la recherche de l'équilibre, en outre celle de l'autonomie, chez l'apprenant. Du point de vue cognitif, nous tenons le fait que «le développement ontogénétique de notre système sensori-moteur est l'illustration même du dynamique cérébral, celui d'un organisme ouvert en perpétuelle recherche de son propre équilibre et de son expression, en échange constant avec l'environnement» (50). À distance, donner des cours à des apprenants haïtiens dont le niveau en français déjà est faible laisse à désirer. Comment l'apprenant arrivera-t-il à développer ce dynamisme vers l'équilibre ? Cette quête d'équilibre participe de l'apport de l'apprenant à sa communauté, il doit en effet être bien acquis par une parfaite réadaptation des pensées, observations, aversions et désirs conscients de l'apprenant : «ces réadaptations organiques rudimentaires, qui sont pour ainsi dire en bourgeon, sont importantes parce qu'elles sont le seul moyen d'échapper à la domination des habitudes routinières et des impulsions aveugles » (Dewey 431). Nous sommes sceptiques que les cours en ligne soient perçus et conçus dans cet état d'esprit, et puissent d'ailleurs en tenir compte. Il importe de noter à la suite des travaux réalisés (Trocmé-Fabre 124) en psychologie que «la structure des méthodes d'éducation, beaucoup plus que leur contenu, influence les processus mentaux, emprisonne la pensée dans un moule et la coule dans le béton des contraintes conceptuelles et perceptuelles. L'intelligence est souvent confondue avec la conformité aux normes et à la capacité d'adaptation au système ». En situation d'apprentissage à distance, l'esprit ne connaît pas toutes ces contraintes comparativement à celle en présentiel, alors que ces contraintes sont importantes pour la recherche de l'équilibre que requiert notre organisme : tout se fait pas l'interaction et des contraintes, par exemple à partir du langage : « [Mais] le langage ne serait pas l'instrument efficace qu'il est, s'il ne se situait pas dans le contexte d'un usage frustre et plus tangible de moyens matériels » (Dewey 73). C'est dans les interactions avec le monde environnant qu'on acquiert efficacement une langue. Il en va de même pour l'acquisition des savoirs et des contenus, d'autant plus que «quand les enfants vont à l'école, ils ont déjà un esprit. Ils ont des connaissances et des aptitudes à juger auxquelles on peut faire appel en utilisant le langage » (73). En fait, tout éducateur soucieux du développement cognitif de ses apprenants, délivrant des enseignements présentiel ou à distance, doit savoir que l'Internet ou les TIC ne peuvent pas remplacer le cerveau humain qui les ont conçus et inventés, et qu'il existe qu'un lien très minime entre la salle de classe et les « cours en ligne ». 


\section{Analphabétisme technoculturel et les jeunes haïtiens non scolarisés}

Nous admettons sans réserve qu' « une personne formée est celle qui sait exécuter les principales tâches qui lui incombent mieux qu'elle ne pourrait le faire sans formation, 'mieux' signifiant avec plus d'aisance, d'efficacité, d'économie, de rapidité, etc. » (Dewey 104). Nous savons d'une part que «le développement de pratiques innovantes modifie l'organisation traditionnelle des situations d'enseignement et d'apprentissage ", et d'autre part que «le recours à des produits informatisés conduit en effet à rompre avec les unités de temps, d'action, de lieu, des formations traditionnelles » (Blamont 99). Nous croyons en outre que les habitudes contribuent au développement cognitif (Dewey) de l'apprenant. Il s'ensuit que la formation à distance, notamment des jeunes, nécessite une double implication : une connaissance adéquate de leur part permettant de manipuler efficacement des outils relatifs aux cours mis en ligne en vue d'une participation active ; une prise de recul de la part des participants par rapport à ses vieilles pratiques (aller sur l'Internet comme un parc d'attraction, un lieu d'audience, de discussion, de divertissement). Par ailleurs, quand certains avancent: «le Net en quête de talents » (Blamont), d'autres prétendraient trouver la solution au problème éducatif haïtien en passant par des cours en ligne sans s'interroger sur le taux d'analphabètes que compte Haïti, le niveau socioéconomique du pays, le nombre de foyers qui n'ont pas d'abonnement Internet ou n'y ont pas accès, le nombre d'analphabètes en technologie dans tout le pays. Nous considérons quelques chiffres disponibles à ce niveau, en vue d'une idée globale sur la réalité haïtienne en nous penchant sur la situation du système éducatif haïtien. Cette situation nous peint un système perturbé par des crises sociopolitiques à un point tel que « le système éducatif haïtien s'est développé dans un environnement marqué par une longue crise sociopolitique de plus d'une vingtaine d'années (1983-2008). $\mathrm{Au}$ cours de cette période, le pays a connu plus de quatorze gouvernements et cinq coup d'état [...] » (GTEF 67). Si nous ajoutons à tout cela la période allant de 2004 à 2007, nous verrons que cette dernière " fut l'émergence et l'intensification d'un climat de violence sans pareil, marqué notamment par des assassinats et des enlèvements aveugles d'hommes, de femmes et d'enfants contre rançons, provoquant ainsi un mouvement important de déplacements de population de certaines zones de non droits par l'État vers d'autres zones estimées moins dangereuses et l'appauvrissement systématique d'une couche importante de la population» (GTEF). Des études (Bernardin) ont montré que «la croissance démographique accélérée (2.05\% par an) entraînant le problème de la dégradation des conditions de vie de la population, la faible productivité agricole, le faible niveau 
d'industrialisation, le taux élevé d'analphabétisme (80\%), le sous-équipement généralisé, l'inégale répartition des revenus ( $1 \%$ de la population concentre $49 \%$ des revenus), le chômage endémique (70\%), la balance commerciale déficitaire (8 138000000 gourdes en 1996 [...]), l'accentuation de la pauvreté et du phénomène de bidonvillisation, sont autant de problèmes inhérents à la nature même du sous-développement ». À la lumière de ces estimations, le recours aux cours en ligne mis non seulement à l'intention des jeunes mais aussi dans l'idée d'atteindre une formation de qualité à la place du système éducatif haïtien ne fera qu'amplifier les échecs en éducation en Haïti. Nous utilisons un tableau que nous devons à Blamont (103) résumant les différents paramètres relatifs aux choix des ressources et des activités ainsi qu'à la création des parcours.

\begin{tabular}{|c|c|c|}
\hline Paramètres & Critères & Indicateurs de mesure \\
\hline & $\begin{array}{ll}\text { Quantité } & \text { d'activités } \\
\text { obligatoire } & \end{array}$ & $\begin{array}{l}\text { Nombre total d'activités obligatoires dans le } \\
\text { cours }\end{array}$ \\
\hline & $\begin{array}{l}\text { Détails de réalisation des } \\
\text { activités }\end{array}$ & $\begin{array}{l}\text { Temps (en jours) accordé pour réaliser et } \\
\text { soumettre les activités obligatoires }\end{array}$ \\
\hline & Consignes des activités & $\begin{array}{l}\text { Spécificité (plus ou moins élevée) des consignes } \\
\text { de réalisation d'activités }\end{array}$ \\
\hline \multirow[t]{2}{*}{ Degré de difficulté } & Difficulté cognitive & Difficulté relative aux prérequis \\
\hline & Charge de travail & $\begin{array}{l}\text { Nombre d'heures de travail hebdomadaire } \\
\text { estimé afin de réussir le cours }\end{array}$ \\
\hline \multirow[t]{2}{*}{$\begin{array}{l}\text { Degré d'interaction entre } \\
\text { pairs }\end{array}$} & $\begin{array}{l}\text { Activités } \\
\text { «formelles» }\end{array}$ & $\begin{array}{l}\text { Importance accordée à la participation aux } \\
\text { échanges de groupes et évaluation par les paris }\end{array}$ \\
\hline & $\begin{array}{l}\text { Activités } \\
\text { «informes » }\end{array}$ & $\begin{array}{l}\text { Importance accordée à la participation aux } \\
\text { échanges synchrones et asynchrones entre } \\
\text { apprenant }\end{array}$ \\
\hline
\end{tabular}

Dans le cas des pays comme Haïti en voie de développement, ce tableau présente un ensemble de points suscitant des contraintes à trois niveaux au moins: financier (les participants dont les moyens ne le leur permettent pas, sont censés se rendre à un cybercafé ou rester sans travailler les activités), logistiques (les participants ne maîtrisant ou manipulant pas bien ou très peu, voire pas du tout la plateforme ou d'autres outils informatiques [lenteur en manipulation de la souris ou du clavier par exemple, qui n'ont aucune connaissance dans le domaine d'Internet]), techniques (problème fréquent ou coupure d'électricité). Tout cela ne concourt pas à la progression curriculaire des participants aux cours en ligne. 


\section{Situation socioéconomique d'Haïti}

Le développement économique de la République d'Haïti, l'augmentation de son indice de développement humain et le changement de son statut de pays le moins avancé de la région passent nécessairement par l'éducation de sa population jeune de tous âges dont les tranches (6-12 ans, 13-18, 19-30, 31-55 et 56 et plus) correspondent respectivement à des populations de 558 163, 175 058, 391 860, 1066 790, 601958 ne sachant ni lire ni écrire), selon GTEF (69), à la suite d'IHSI (2005) $)^{3}$. S'y ajoutent des couches moins jeunes de la population qui, par leur travail dans les divers secteurs formels et informels, constituent la force vive qui alimente le produit national brut, d'où le grand enjeu que constitue l'accès à l'éducation pour tous les niveaux. Des indices le confirme : «le problème de la dégradation des conditions de vie de la population, la faible productivité agricole, le faible niveau d'industrialisation, le taux élevé d'analphabétisme (80\%), le sous-équipement généralisé, l'inégale répartition des revenus ( $1 \%$ de la population concentre $49 \%$ des revenus), le chômage endémique (70\%), la balance commerciale déficitaire (8 138000 gourdes en 1996), l'accentuation de la pauvreté et du phénomène de bidonvilisation » (Bernardin 3). De plus, en termes d'exportation, des études (GTEF 68) ont montré qu' « en 2002-2003, le pays a exporté seulement $13 \%$ de son produit intérieur brut alors qu'il est dépendant des importations pour nourrir sa population ». En fait, « de la fin des années soixante-dix à 2005, la croissance économique réelle a été en moyenne de $0.31 \%$ l'an ». D'autres chiffres (GTEF 68) ont montré qu' «après avoir bénéficié d'une certaine stabilité économique au cours des années soixante-dix, la situation s'est par la suite considérablement dégradée. L'économie haïtienne est entrée dans une période de récession et de son contreperformance généralisées dès 1980$1981 » ;$ «au cours des cinq exercices budgétaires 2001-2006, le pourcentage du budget national consacré à l'éducation est passé d'environ $17 \%$ à $10 \%$ et la majeure partie $(85 \%$ en moyenne) a été destinée au fonctionnement [...]»(68). Ce coup d'œil panoramique sur la situation socioéconomique du pays nous montre clairement que les initiatives des cours en ligne sont conçues notamment pour les jeunes dont les parents possèdent les moyens financiers nécessaires dans un pays où règnent «l'inégale répartition des revenus $(1 \%$ de la population concentre $49 \%$ des revenus), le chômage endémique (70\%) (Bernardin).

\section{Situation sociolinguistique ${ }^{4}$ haïtienne et cours en ligne}

Si présenciellement et en temps réel, par rapport à la question de langue constituant un des problèmes majeurs du système éducatif haïtien, l'enseignement/apprentissage des 
disciplines non linguistiques s'avère complexe, former des jeunes haïtiens à distance pour le pays le sera davantage. En effet, l'idée de mettre des cours en ligne en s'imaginant qu'une telle initiative va constituer un moyen certain permettant d'aboutir à la transformation de la société haïtienne au plan éducatif (formation de qualité) ${ }^{5}$ par le biais de ces cours s'avère utopique. Paradoxalement, il faut noter qu'en dépit du fait qu'on estime à 10\% (Rey) le nombre d'Haïtiens qui parlent français, la tendance est que « chez les jeunes, la valorisation du créole n'est pas évidente malgré les acquis que la langue a enregistrés dans le domaine de la vie politique » (Saint-Germain 611). Par exemple, un cours de géométrie ${ }^{6}$ exige de la part de l'apprenant un minimum de français ${ }^{7}$ pour la compréhension et l'appropriation des concepts employés dans ce domaine. Face à de telles situations complexes, pour les cours en ligne proposes, il y a de nombreux défis à relever qui viennent s'ajouter aux défis liés au contexte éducatif haïtien. Mais il est d'actualité»: «de nombreux discours communs présentent les technologies d'information et de communication (TIC) comme susceptibles de transformer les pratiques d'enseignement/d'apprentissage, voire les modes d'accès à la connaissance » (Siméone 2). D'emblée, les défis à relever dans le pays sont énormes, et nous présupposons que les élèves inscrits à ces cours en ligne auront de grandes difficultés tant au plan linguistique, logistique et matériels de travail que pédagogique ${ }^{8}$, ce qui leur empêchera du coup de profiter de ces cours mis à leur disposition ou intention. Concernant les cours à distance mis en ligne dans la langue française, tout enseignant ou éducateur conscient de la situation sociolinguistique haïtienne sait pertinemment que les mathématiques ou la géométrie, ou mieux encore la trigonométrie, exigent, à ce point de vue, une aisance ou une compétence suffisante en français de la part de l'apprenant pour s'en approprier les concepts ou notions liées à chacun de ces domaines. Or, le français est la langue utilisée par les concepteurs des cours en ligne dédiés à des jeunes, dont les parents n'ont pas de moyens suffisants, ou très peu, pour en assurer la scolarité. Or, la situation sociolinguistique haïtienne existe telle qu' « il y a une minorité linguistique constituée de bilingues, une minorité urbaine en opposition à la majorité rurale, une minorité riche par rapport à une majorité pauvre, etc. L'un des paramètres sur lequel est fondé ce clivage social est la maîtrise de la langue » (SaintGermain 611). Le français, comme toute langue, est un « vaste système de structures, différent de celui des autres langues, dans lequel sont ordonnées culturellement les formes et les catégories par lesquelles l'individu non seulement communique mais aussi analyse la nature, aperçoit ou néglige tel ou tel type de phénomènes et de relations, dans lesquelles il coule sa façon de raisonner, et par lesquelles il construit l'édifice de sa connaissance du 
monde ». Par ailleurs, des travaux (Déjean 41) ont montré que le créole est le « sèl prensipal mwayen pou plis pase 9 milyon Ayisyen. Aprann yon lòt lang, tankou franse, se yon bon bagay, men sa pa kab rive fèt pou tout popilasyon yon peyi ki deja gen yon lòt lang » ${ }^{9}$. En fait, selon une hypothèse (voir Sapir-Whorf), les modes de pensées sont déterminés par la langue maternelle. Un modèle de cours de trigonométrie mis en ligne par l'École haïtienne sans frontières peut à lui seul confirmer la situation et nous en donner une nette idée. Ainsi, nous remarquerons particulièrement que le souci de l'informateur est de conduire ses apprenants à retenir les notions en trigonométrie. Les cours mis en ligne sont contraires à la réalité sociolinguistique du pays en ce sens que la langue utilisée dans le cadre de ces cours est le français maitrisé par 10\% de la population. Selon l'UNESCO, le meilleur outil auquel un système éducatif doit recourir dans l'enseignement, c'est la langue parlée par toute la population d'un pays. Pour le cas d'Haiti, le «kreyòl se sèl mwayen prensipal konesans pou plis pase nèf (9) milyon moun ann Ayiti ${ }^{10} \gg$ (Déjean 42). En accord avec cette évidence, les cours mis en ligne à l'intention des jeunes dont les parents n'ont pas de moyens leur permettent de fréquenter un établissement scolaire, devraient prendre en compte le statut social des jeunes qui n'ont que le créole et qui ne sont pas en contact direct avec le français dans leur quotidien. De récentes études ont montré que « youn nan raison ki lakòz sistèm lekòl Ayiti a fè fayit, se paske lang yo plis itilize pou yo anseye elèv yo se franse alòske pi fò Ayisyen - kit se elèv, kit se pwofesè - se kreyòl sèlman yo pale byen (Gen petèt 500000 Ayisyen ki pale franse divès degre pami 10 milyon Ayisyen ann Ayiti) $»^{11}$ (Degraff 133). Comme cela a été recommandé ${ }^{12}$, «tout enfant naît dans un certain milieu culturel ; la langue fait partie de ce milieu en même temps qu'elle l'exprime. Ainsi, l'acquisition de cette langue (sa 'langue maternelle') s'insère dans le processus par lequel l'enfant assimile le milieu culturel ». Les participants à titre d'apprenants qui ont déjà le créole, ont déjà acquis la langue créole, mais aussi n'ont eu leurs premières années de scolarisation en créole (UNESCO ; Réforme Bernard) en vue de leur initiation au français. En effet, l'acquisition des notions mathématiques à intégrer en français s'avérera difficile. Une étude montre que les élèves haïtiens font face à d'énormes difficulté quand il s'agit de l'enseignement des disciplines telles les mathématiques : «[...] malgre nou pa pwofesè lekòl, nan mezi mwayen nou pèmèt nou, nou pa janm sispann louvri je moun nan sosyete a sou pwoblèm timoun yo nan aprantisaj matematik [...], si gen difikilte pou elèv, gen difikilte tou pou pwofesè yo $»^{13}$ (Chéry 252). À la lumière de ce constat en relation avec les recommandations formulées dans (UNESCO ; Réforme Bernard), nous déduisons que les élèves haïtiens feront face à des 
difficultés, car l'enseignement des disciplines non-linguistiques se fait dans une langue que la majorité des apprenants ne maîtrisent pas. De fait, les difficultés que rencontrent les élèves qui suivent des cours en ligne (assistés ou non par un guide pédagogique), augmenteront. Un des aspects sur lequel met accent un professeur des cours en ligne c'est la rétention. En effet, « la manière dont les enseignants enseignent doit être au centre de toute réforme destinée à améliorer la qualité » (UNESCO 172) (cité par Gurqua et Gauthier). Par ailleurs, « the role played by Haitian Creole (HC) - spoken by the entire country's population - versus that of French - mastered only by a small minority - constitutes the central issue in the educational system of Haiti. In 1979, the Réforme Bernard was instituted, declaring HC the primary language of instruction during the first four years of elementary education $»^{14}$. De surcroit, dans un rapport ${ }^{15}$ sorti en juillet 2006 , le créole y est perçu comme une langue qui devrait occuper une place privilégiée dans l'enseignement, elle doit être étudiée en tant que langue et servir de langue d'enseignement du cycle préscolaire à l'université. Notons qu' « un être dont les activités sont liées à celles d'autrui, a un environnement social » (Dewey 49). Cet environnement est un prisme à travers un langage partagé par l'ensemble des membres d'une communauté partage l'environnement. Quel système éducatif peut-il prétendre ne pas tenir compte de cette évidence? On apprend mieux et on résout mieux les problèmes en interaction à partir de la langue maîtrisée et parlée par cette communauté : «le langage est [...] un cas où notre propre action et celle de quelqu'un d'autre renvoient à une situation commune. D'où sa portée sans pareille comme moyen de direction sociale » (73). Les participants au cours en ligne qui ne parlent pas français, pourront bénéficier des cours mis en ligne, et l'interaction, s'il y en a, avec des participants parlant cette langue ne leur sera pas possible, ce qui nous renvoie à la question de normes en sociolinguistique, dites normes endogène/exogène. Il existe deux langues en Haïti, créole et française, parlées respectivement par toute la population du pays et par une portion de la population. Paradoxalement, le français est la langue des cours mis en ligne la plus présente comme cela se faisait avant l'officialité du créole en 1987.

\section{Les Nouvelles Technologies de l'Information au service de l'homme mais restent coûteuses}

Au-delà des préoccupations d'ordre linguistique, d'autres préoccupations liées à la situation socioéconomique sont à prendre en compte. En Haïti, des études ont montré que la majorité de la population vit en dessous du seuil de la pauvreté absolue et, à ce propos, «la 
Banque Mondiale 'estime aujourd'hui que $80 \%$ des deux-tiers de la population qui vivent en zone rurale sont des pauvres' ». Ce constat, qui est un fait réel et manifeste de jour en jour, nous rend très sceptiques quant à la possibilité pour les élèves inscrits d'accéder régulièrement à la plateforme et suivre au temps voulu les cours en ligne. Noël dresse en ce sens un bon rapport de la réalité des TIC en Haïti : «l'extension d'applications NTIC suppose l'universalisation en amont de l'accès et du droit à la communication. La tâche n'est pas facile, tant sont grandes les disparités entre les groupes et les régions du pays. Haïti fait en effet partie du lot de pays dont les structures en place ont longtemps gardé et gardent encore isolées des couches de la population » (14). En tenant compte des coûts que peut exiger une formation à distance en Haïti à l'heure actuelle, il y a lieu de mettre en question non seulement sur la qualité de la formation à distance, mais aussi la progression des contenus. Par exemple l'inscription aux cours en ligne est facile : un intéressé peut demander à un cousin, à une sœur ou un frère, un ami, une proche de l'inscrire à la plateforme où l'on donne des cours, ou entrer dans un cyber pour l'inscription. Mais lorsqu'il s'agit de rester en constant contact avec l'Internet, le bât blesse. Combien de familles haïtiennes possèdent-elles un ordinateur? Combien de parents qui peuvent payer un abonnement d'internet chez eux, en Haïti ? Combien de jeunes actifs qui travaillent et qui ont un salaire ? Combien de jeunes qui ont une connaissance de base en informatique ? Ou du moins, combien d'entre eux qui ont eu un cours d'initiation à l'Internet ? Point n'est besoin de répondre à ces importantes interrogations. Il suffit de vivre la situation socioéconomique du pays pour être convaincu que « le taux élevé d'analphabétisme (80\%), le sous-équipement généralisé, l'inégale répartition des revenus ( $1 \%$ de la population concentre $49 \%$ des revenus), le chômage endémique $(70 \%) »$ de la difficulté de semblables projets (Bernardin). Le pouvoir d'achat de chaque Haïtien ne lui permet pas de se payer le luxe d'un ordinateur, voire payer un abonnement d'Internet. En Haïti, en 2006 par exemple, avoir accès ${ }^{16}$ à Internet exigeait une somme de deux-cents dollars étatsuniens pour l'achat d'un modem, et de trente-neuf dollars par mois pour l'abonnement. A l'heure actuelle, la situation n'a pas changé : le prix mensuel de l'abonnement varie d'un fournisseur à l'autre. Certains fournisseurs exigent d'un client entre trente jusqu'à cent quatre-vingt-cinq dollars étatsuniens le mois pour un abonnement. 


\section{Enjeux, défis, perspectives}

Dans cette partie, nous analysons quelques enjeux et défis que nous supposons liés à la formation à distance, notamment aux cours en ligne destinés aux jeunes haïtiens. Nous dégagerons quelques perspectives et conclurons.

\section{Les enjeux}

Les programmes de promotion de scolarisation universelle et de l'éducation pour tous peinent encore à assurer que tous les enfants d'âge scolaire fréquentent l'école et s'y maintiennent pour développer au mois les compétences de l'Éducation de base. Les élèves du secondaire reçoivent à $90 \%$ leur enseignement dans des institutions non publiques de qualité variée, tandis que les $10 \%$ qui sont admis dans les lycées disséminés à travers les dix départements géographiques du pays, y sont agglutinés dans des classes en général pléthoriques dans la quête du savoir qui devrait les amener à l'obtention du baccalauréat et faciliter leur accès au marché de l'emploi auquel la formation ne les a guère préparés à l'université. Cette dernière, privée ou publique, accommode un faible pourcentage du nombre restreint des bacheliers admis. Il en résulte à tous les niveaux un gaspillage de cerveaux et des déficits de plus en plus grands à combler. Au cours des trois dernières décennies, le système éducatif haïtien a fait l'objet de nombreux diagnostics techniques qui ont mis en évidence ses indiscutables forces ainsi que ses trop nombreuses faiblesses. En réponse, ont été successivement initiés jusqu'à date des temps forts de régulation et des tentatives de mettre en œuvre la Réforme Éducative de 1982, conçue pour apporter des améliorations consistantes et durables aux faibles performances du système autant dans les conditions de scolarisation des élèves et la qualité de leurs apprentissages que dans celle de l'instruction dispensée par les enseignants à tous les niveaux. Ces efforts se sont effectués sur un fond de turbulences politiques sporadiques, causées par la quête nationale incessante et douloureuse d'un fonctionnement démocratique approprié pour Haïti. À cette situation d'instabilité récurrente se sont ajoutés, durant la même période et avec une fréquence déconcertante, le passage de cyclones meurtriers, les épisodes d'inondations et autres catastrophes environnementales dont la plus récente, le tremblement de terre de janvier 2010. Ils ont tous eu des incidences malheureuses sur le fonctionnement régulier des écoles et sur le développement dans différentes générations d'élèves des compétences académiques et de savoir-faire socioculturels et technologiques prévus dans leurs programmes d'études. Cette situation qui semble sans issue, a fait naître l'idée que la formation numérique grâce aux cours en ligne, notamment pour le cycle d'études secondaire, pouvait être une solution viable pour remettre à 
l'honneur des apprentissages de qualité au sein du système éducatif. L'analyse de cette proposition permet de mettre en lumière les défis à relever pour le succès d'une telle initiative.

Défis

La considération d'une telle initiative s'inspire évidemment d'une vue considérablement réductrice de l'éducation assimilée à l'absorption par les populations-cibles d'un volume de savoirs codifiés et encapsulés dans un certain nombre de modules disciplinaires numériques. Il convient de mettre en évidence les défis énormes auxquels doit faire face la réalisation de cet enseignement à distance. A travers ce modèle promu, sont largement sous-estimées les caractéristiques liées aux apprenants et qui affectent négativement leurs apprentissage : la grande diversité de leurs profils académiques et linguistiques, la variété de leurs styles d'apprentissage (tendances à la mémorisation et manque d'autonomie et de sens de responsabilité ), leurs motivations psychologiques et représentations socioculturelles ainsi que leur inégalité d'accès aux moyens économiques de participation aux conditions d'apprentissage en ligne telles que la disponibilité d'un ordinateur, l'accès régulier à des sources d'énergie électrique et l'accès continu à une connexion d'Internet à débit stable. Sont également minimisées les pesanteurs de la planification et de la gestion logistiques ainsi que les exigences qu'impose le respect des normes qualitatives régissant la conception de la structure pédagogique des module de cours, la mise en ligne des cours ainsi que l'encadrement humain à travers la progression des activités d'apprentissage. A noter que la résistance à l'utilisation du créole comme outil retarde aussi l'efficacité de la formation en ligne quand l'objet n'est pas le français ou une autre langue. L'existence et le fonctionnement d'un cadre légal de régulation, d'encadrement et de validation de cette forme d'éducation en ligne par l'autorité compétente constituent souvent un autre point faible et même névralgique de ces innovations, comme il en est d'ailleurs le cas, jusqu'à date, pour l'utilisation des nouvelles technologies de la communication au niveau national.

Est-ce à dire que l'éducation en ligne n'est pas réalisable efficacement en Haïti ? A quelles conditions l'utilisation des TIC en éducation en Haïti peut-elle être bénéfique à la qualité de la population ? Il importe de souligner, après tant d'autres, que la tendance à privilégier le médium et les contenus au détriment de la réflexion continue sur les valeurs liées au développement humain et sur l'organisation pédagogique interne des documents de formation dessert considérablement la qualité de l'éducation par les TIC. Elle table sur une 
autonomie d'apprentissage quasi-inexistante, rendue difficile par les faibles compétences linguistiques des apprenants, tant qu'elle se réalise dans une langue qu'ils ne comprennent pas.

\section{Perspectives}

Il est indéniable que la pénétration accélérée et continue de la téléphonie mobile dans les dix départements du pays, l'avènement et la démocratisation du coût de nouvelles générations de supports électroniques à l'apprentissage (téléphones intelligents et tablettes) et la mise au point de systèmes de plus en plus performants pour la disponibilité à moindre coût des énergies renouvelables, notamment le solaire, constituent des améliorations notables des conditions de l'utilisation fructueuse du numérique au service de l'éducation en Haïti.

Il importe aujourd'hui de privilégier la structuration de communautés apprenantes et leur réflexion continue partagée autour des savoirs qu'elles acquièrent, des valeurs qui donnent du sens à leur vie et à leurs apprentissages pendant qu'elles bénéficient pleinement de la structuration de leur pensée grâce à la rigoureuse organisation pédagogique des modules de cours de formation en ligne.

En première ligne des besoins nationaux se trouvent les enseignants de tous les niveaux. La formation par les TIC serait hautement bénéfique, autant pour leur mise à niveau académique que pour l'acquisition des savoir-faire pédagogiques et didactiques nécessaires à la compréhension et à l'exercice adéquat de leur profession. Le rôle qu'ils jouent à l'école et leur position privilégiée d'observateurs et d'interlocuteurs des jeunes leur permettent de considérer les valeurs et tendances au sein de leur société. En groupes d'écoles d'une même région ou d'un même quartier, ils sont à même de développer une réflexion partagée en communauté d'apprentissage, autant en interaction sur Internet qu'en situation de regroupements périodiques en présentiel. Ce premier choix est aussi une stratégie payante pour l'avenir, car il garantirait la disponibilité d'un meilleur encadrement des jeunes générations dans l'utilisation éducative des TIC, grâce à la présence d'une masse critique d'enseignants formés pédagogiquement à la réflexivité sur les valeurs éducatives et sociales et aux TIC. Une intégration rationnelle et efficace des TICs à l'échelle nationale est à ce prix. Elle devient, en effet, une nécessité dans la mesure où elle peut permettre de réduire l'analphabétisme et d'accompagner tous les publics-cibles actifs dans l'économie formelle et informelle d'Haïti. 


\section{Références bibliographiques}

Bernardin, E .A. « La planification régionale en Haïti ». 1999. http://cybergeo.revues.org/4840\#article-4840 consulté le 13/03/2015 .

Blamont, J. Pour une révolution de l'évolution en Haïti. Port-au-Prince : U d'État d'Haïti, 2013.

Dewey, J. Démocratie et éducation. Tr. Gérard Dedalle. Paris : Amand Colin, 1990.

Degraff, M. Ayiti pare/« Haiti is ready ». 2013. https://www.youtube.com/watch?v=HJ6kx7JX24U ? consulté le 01/04/2015.

École haïtienne sans frontières. https://www.youtube.com/watch?v=24Q12O7ScD4

Godinet, H. «Scénario pour apprendre en collaborant à distance: contraintes et complexité ». Le campus numérique FORSE, pistes pour l'ingénierie de la formation à distance. Dir. J. Wallet. Mont-Saint-Aignan : PURH, 2007.

Govain, R., dir. «Ak Kòlòk Entènasyonal sou Akademi Kreyòl Ayisyen an 26-29 oktòb 2011 ». Akademi Kreyòl Ayisyen : Ki wòl ? Ki avantaj ? Ki defi ? Ki avni ? Port-auPrince : U d'État d'Haïti, 2011.

Gurqua, A et C. Gauthier. «Suggérer à demi-mot. À propos du discours pédagogique de l'UNESCO et de l'OCDE ». 2012.

http://www.ibe.unesco.org/Countries/WDE/2006/LATIN_AMERICA_and the_CARI BBEAN/Haiti/Haiti.pdf Consulté le 11/09/2015.

Hess, F. M. et B. Saxberg. Breakthrough leadership in the digital age: Using Learning science to reboot schooling. Np : Corwin, 2014.

GTEF. «Pour un Pacte national sur l'Éducation en Haiti. Rapport au Président de la République ». Port-au-Prince : Haïti : Port-au-Prince, 2010.

Noël, J.M.R. L'évolution des technologies de l'information en Haïti (1995-2010) : entre l'indécision politique et le flou juridique. Port-au-Prince : U d'État d'Haïti, 2014.

Rey, Alain. 2010. http://www.tv5monde.com/cms/chaine-francophone/lf/Tous-les-dossierset-les-publications-LF/Dictionnaires-et-francophonie/Les-videos/p-11647-Alain-ReyFrancophonie-etat-des-lieux.htm Consulté le 28/02/2015.

Saint-Germain, M. (1997). «Problème linguistique en Haïti et réforme éducative : quels constats ? ». Revue des sciences de l'éducation 23.3 (1997) : 611-642.

Trocmé-Fabre, H. J'apprends, donc je suis, 3e éd. Paris : Organisation, 1997. 


\footnotetext{
NOTES

${ }^{1}$ https://www.youtube.com/watch?v=Qes8CuMpXM8

2 AFNOR = Association Française de Normalisation) $($ voir

http://www.utc.fr/ tthomass/Themes/Unites/unites/infos/normes/Les_normes.pdf) consulté le 04/05/2015

${ }^{3}$ IHSI (2005). Résultats définitifs du $4{ }^{\text {ème }}$ recensement général de la population

${ }^{4}$ Ayiti pare (en anglais, « Haiti is ready » https://www.youtube.com/watch?v=HJ6kx7JX24U

${ }^{5}$ École haïtienne sans frontières https://www.youtube.com/watch?v=24Q12O7ScD4

${ }^{6}$ École haïtienne sans frontières, (2013). Cercle trigonométrique : sinus et cosinus https://www.youtube.com/watch? $\mathrm{v}=4-$ TEsEg0rb4 consulté le $1^{\text {er }}$ avril 2015

${ }^{7}$ Il ne s'agit pas du français général mais d'un français spécifique ou référentiel.

8 École haïtienne sans frontières. Cercle trigonométrique: sinus et cosinus https://www.youtube.com/watch?v=4-TEsEg0rb4

consulté le $1^{\mathrm{er}}$ avril 2015. (Voir également : Fonctions cosinus et sinus / Placer des points sur un cercle trigonométrique (2))

${ }^{9} \mathrm{~L}$ '« unique principal moyen pour plus de 9 millions d'haïtiens. Apprendre une autre langue, comme français, est une bonne chose, mais cela ne peut pas se faire pour toute la population d'un pays qui a déjà une langue », notre traduction.

10 « [le] créole est le seul principal moyen de connaissances pour plus de neuf million d'habitants », notre traduction.

${ }^{11}$ L'une des raisons qui est occasionnent les échecs du système éducatif haïtien, c'est le fait que ce n'est pas la langue la plus utilisée dans l'enseignement c'est le français tandis que la majorité des Haïtiens - élèves ou professeurs - parlent uniquement mieux le créole (il existe peut-être 500000 Haitiens qui parlent français de divers degrés parmi les 10 millions d'Haïtiens en Haïti).

12 UNESCO (1953). L'emploi des langues vernaculaires dans l'enseignement http://unesdoc.unesco.org/images/0013/001315/131582fo.pdf consulté le 03/04/15

13 «En dépit du fait que nous ne sommes pas professeur d'école, nous n'avons jamais cessé d'ouvrir les yeux des gens dans la société sur les obstacles que rencontrent les enfants dans l'apprentissage des mathématiques [...], si les problème existent pour les élèves, il en va de même pour les professeurs ».

${ }^{14}$ «Le rôle joué par le créole haïtien (HC) - parlé par toute la population du pays - par rapport au rôle que joue le français maîtrisée par une petite minorité seulement - constitue la question centrale dans le système éducatif haïtien. En 1979, la Réforme Bernard a été institué, en déclarant que le HC, la langue principale d'enseignement pendant les quatre premières années de l'enseignement primaire ». http://www.indiana.edu/ clacs/events/docs/1415docs/valdman-flyer.pdf consulté le $\underline{03 / 04 / 2015}$

${ }^{15}$ Haiti-International Bureau of Education - UNESCO, (2006).

http://www.ibe.unesco.org/Countries/WDE/2006/LATIN_AMERICA_and_the_CARIBBEAN/Haiti/Haiti.pdf

16 Le nouvelliste (2006), http://lenouvelliste.com/lenouvelliste/article/33202/Le-Net-Mobile-de-la-mobilite-pour-lesconnexions-internet/consulté le 02/04/2015
} 